\title{
Studies of Coumarins from the Chinese Drug Qianhu, XXVII'): Structure of a New Simple Coumarin Glycoside from Bai-Hua Qianhu, Peucedanum praeruptorum
}

\author{
Hirotaka IshII, ${ }^{2)}$ Yoshihito OKadA, Masaki BaвA, and Toru OKuYAмA* \\ Department of Natural Medicine and Phytochemistry, Meiji Pharmaceutical University; 2-522-1 Noshio, Kiyose, Tokyo \\ 204-8588, Japan. Received May 9, 2008; accepted June 23, 2008; published online June 25, 2008
}

\begin{abstract}
A new simple coumarin glycoside, named praeroside VI (1), along with six known coumarins, were isolated from an aqueous extract of Bai-Hua Qianhu, the root of Peucedanum praeruptorum DunN. (Umbelliferae). Spectroscopic analysis and chemical studies were carried out to investigate the structure of the new coumarin, which was concluded to be 1 . Additionally, two simple glycosidic coumarins and four simple nonglycosidic coumarins were identified as apiosylskimmin (2), hymexelsin (3), umbelliferone (4), scopoletin (5), isofraxidin (6) and 8-carboxy-7-hydroxy coumarin (7), respectively. This is the first reported identification of compound 7 as a constituent of plant materials.
\end{abstract}

Key words simple coumarin; Bai-Hua Qianhu; praeroside VI; Peucedanum praeruptorum

The traditional Chinese medicine, Qianhu, which consists of the roots of Peucedanum praeruptorum DunN. and $P$. decursivum Mахім. (Umbelliferae), is used for treatment of respiratory disease and pulmonary hypertension. In the course of our search for the active ingredients of Qianhu, we have reported the structures and biological activities of coumarins isolated from both plants. We isolated the angular non-glycosidic pyranocoumarins $\mathrm{Pd}-\mathrm{Ia}, \mathrm{Pd}-\mathrm{Ib}, \mathrm{Pd}-\mathrm{II}$ and $\mathrm{Pd}-$ III, and the angular glycosidic pyranocoumarins praeroside II, praeroside III, praeroside IV and praeroside V, along with linear glycosidic furocoumarin praeroside I, from Bai-Hua Qianhu (Peucedanum praeruptorum DunN.), and the linear non-glycosidic pyranocoumarins Pd-C-I, Pd-C-II, Pd-C-III, Pd-C-IV, Pd-C-V and AD-I, as well as various saponins, from Zi-Hua Qianhu, the root of $P$. decursivum Maxim. ${ }^{3-22)}$ In addition, we recently isolated two novel angular pyranocoumarin glycosides, peucedanosides $\mathrm{A}$ and $\mathrm{B}$, along with apterin $^{23)}$ and praerosides $\varepsilon$ and $\phi,^{24)}$ from Peucedanum praeruptorum DunN.

In this paper, we report the isolation and structure elucidation of a new simple coumarin glycoside and six known simple coumarins from Bai-Hua Qianhu collected in China.

\section{Results and Discussion}

Praeroside VI (1) was isolated as a colorless powder, mp $130.5-132.5^{\circ} \mathrm{C}$ (decomp.), $[\alpha]_{\mathrm{D}}+1.0^{\circ}$ (MeOH). The molecular formula $\mathrm{C}_{20} \mathrm{H}_{26} \mathrm{O}_{10}$ was determined by high-resolution (HR) EI-MS, $m / z$ 426.1525 ([M] ${ }^{+}$, Calcd 426.1526). A UV absorption at $326 \mathrm{~nm}$ indicated the presence of a coumarin nucleus. The mass fragmentation pattern $(\mathrm{m} / \mathrm{z} 408,368,301$, $293,264,246,229,205,188,176,147,131)$ was in agreement with the proposed structure $\mathbf{1}$.

The ${ }^{1} \mathrm{H}-\mathrm{NMR}$ spectrum of $\mathbf{1}$ (Table 1) showed two pairs of doublets at $\delta 6.17$ and $7.81 \mathrm{ppm}($ each $1 \mathrm{H}, \mathrm{d}, J=9.5 \mathrm{~Hz}$ ) and at $\delta 7.34(1 \mathrm{H}, \mathrm{d}, J=8.5 \mathrm{~Hz})$ and $6.81 \mathrm{ppm}(1 \mathrm{H}, \mathrm{d}, J=8.3 \mathrm{~Hz})$ which were assigned to $\mathrm{H}-3, \mathrm{H}-4, \mathrm{H}-5$ and $\mathrm{H}-6$ in the coumarin skeleton. The ${ }^{1} \mathrm{H}-\mathrm{NMR}$ spectrum also showed the presence of one hydroxyl group on a C-7 and C-8-substituted isopentenyl simple coumarin glycoside fragment, representing a C-8-(2',3'-dihydroxy-3'-methylbutyl)-coumarin. The characteristic signal was at $\delta 4.06(1 \mathrm{H}, \mathrm{dd}, J=6.7,5.5 \mathrm{~Hz})$ owing to the methine proton at $\mathrm{H}-2^{\prime}$ with an $O$-functional group, which is linked to the methylene groups with signals at $\delta 3.18 \mathrm{ppm}(1 \mathrm{H}, \mathrm{dd}, J=14.5,7.3 \mathrm{~Hz})$ and $\delta 3.14 \mathrm{ppm}(1 \mathrm{H}$, $\mathrm{dd}, J=14.2,5.2 \mathrm{~Hz}$ ). In the sugar proton regions, a doublet signal at $\delta 4.15 \mathrm{ppm}(1 \mathrm{H}, \mathrm{d}, J=7.3 \mathrm{~Hz})$ was assigned as an anomeric proton of D-glucopyranoside linked to the hydroxyl group at $\mathrm{C}-2^{\prime}$. In addition, the large coupling constant $(J=7.3 \mathrm{~Hz})$ of the anomeric proton revealed a $\beta$-linkage in $\mathbf{1}$.

These functional groups were also identified by ${ }^{13} \mathrm{C}-\mathrm{NMR}$ spectroscopy. In distortionless enhancement by polarization transfer (DEPT) experiments, 1 showed eleven oxygenated carbons, including six oxymethines at $\delta 103.46$ (d, G-1), 85.94 (d, C-2'), 77.84 (d, G-5), 77.67 (d, G-3), 74.87 (d, G2), 71.37 (d, G-4) and one oxymethylene at $\delta 62.49$ (t, G-6), respectively (see Table 1). Enzymatic hydrolysis of 1 by cellulase afforded an aglycone (1a), $[\alpha]_{\mathrm{D}}+46.0^{\circ}(\mathrm{MeOH})$, which was identified as $\left(2^{\prime} R\right)-7$-hydroxy-8-(2',3'-dihydroxy$3^{\prime}$-methylbutyl)-coumarin ${ }^{25-27)}$ with D-glucose.
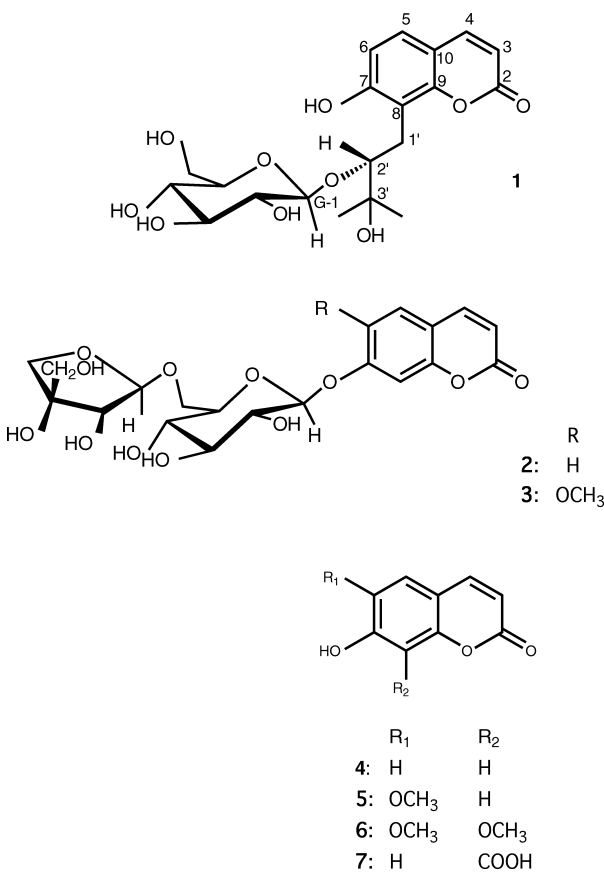

(C) 2008 Pharmaceutical Society of Japan 
Table 1. ${ }^{1} \mathrm{H}$ - and ${ }^{13} \mathrm{C}-\mathrm{NMR}$ Spectral Data of Compounds $\mathbf{1}$ and $\mathbf{1 a}$ in $\mathrm{CD}_{3} \mathrm{OD}$

\begin{tabular}{|c|c|c|c|c|}
\hline & \multicolumn{2}{|c|}{${ }^{1} \mathrm{H}$} & \multicolumn{2}{|c|}{${ }^{13} \mathrm{C}$} \\
\hline & 1 & $1 \mathbf{a}$ & 1 & $1 \mathbf{a}$ \\
\hline 2 & & & $163.28(\mathrm{~s})$ & $163.58(\mathrm{~s})$ \\
\hline 3 & $6.17(1 \mathrm{H}, \mathrm{d}, J=9.5)$ & $6.18(1 \mathrm{H}, \mathrm{d}, J=9.5)$ & $112.10(\mathrm{~d})$ & $111.67(\mathrm{~d})$ \\
\hline 4 & $7.81(1 \mathrm{H}, \mathrm{d}, J=9.5)$ & $7.84(1 \mathrm{H}, \mathrm{d}, J=9.5)$ & $146.08(\mathrm{~d})$ & $146.31(\mathrm{~d})$ \\
\hline 5 & $7.34(1 \mathrm{H}, \mathrm{d}, J=8.5)$ & $7.34(1 \mathrm{H}, \mathrm{d}, J=8.5)$ & $128.20(\mathrm{~d})$ & $127.97(\mathrm{~d})$ \\
\hline 6 & $6.81(1 \mathrm{H}, \mathrm{d}, J=8.3)$ & $6.84(1 \mathrm{H}, \mathrm{d}, J=8.5)$ & $113.55(\mathrm{~d})$ & $113.92(\mathrm{~d})$ \\
\hline 7 & & & $160.37(\mathrm{~s})$ & $161.12(\mathrm{~s})$ \\
\hline 8 & & & $114.32(\mathrm{~s})$ & $115.30(\mathrm{~s})$ \\
\hline 9 & & & $155.11(\mathrm{~s})$ & $154.88(\mathrm{~s})$ \\
\hline 10 & & & $113.38(\mathrm{~s})$ & $113.24(\mathrm{~s})$ \\
\hline $1^{\prime} \mathrm{a}$ & $3.14(1 \mathrm{H}, \mathrm{dd}, J=14.2,5.2)$ & $3.18(1 \mathrm{H}, \mathrm{dd}, J=13.9,2.4)$ & $26.11(\mathrm{t})$ & $26.33(\mathrm{t})$ \\
\hline $1^{\prime} \mathrm{b}$ & $3.18(1 \mathrm{H}, \mathrm{dd}, J=14.5,7.3)$ & $2.92(1 \mathrm{H}, \mathrm{dd}, J=13.9,10.3)$ & & \\
\hline $2^{\prime}$ & $4.06(1 \mathrm{H}, \mathrm{dd}, J=6.7,5.5)$ & $3.67(1 \mathrm{H}, \mathrm{dd}, J=10.3,2.4)$ & $85.94(d)$ & $79.39(\mathrm{~d})$ \\
\hline $3^{\prime}$ & & & $73.81(\mathrm{~s})$ & $73.86(\mathrm{~s})$ \\
\hline \multirow{2}{*}{ gem- $\left(\mathrm{CH}_{3}\right)_{2}$} & $1.22(3 \mathrm{H}, \mathrm{s})$ & $1.29(3 \mathrm{H}, \mathrm{s})$ & $24.70(q)$ & $25.42(q)$ \\
\hline & $1.31(3 \mathrm{H}, \mathrm{s})$ & $1.30(3 \mathrm{H}, \mathrm{s})$ & $26.27(\mathrm{q})$ & $25.49(\mathrm{q})$ \\
\hline G-1 & $4.15(1 \mathrm{H}, \mathrm{d}, J=7.3)$ & & $103.46(d)$ & \\
\hline $\mathrm{G}-2$ & $3.15(1 \mathrm{H}, \mathrm{dd}, J=9.2,7.4)$ & & $74.87(\mathrm{~d})$ & \\
\hline G-3 & $3.18(1 \mathrm{H}, \mathrm{dd}, J=9.2,8.6)$ & & $77.67(\mathrm{~d})$ & \\
\hline $\mathrm{G}-4$ & $3.26(1 \mathrm{H}, \mathrm{dd}, J=9.2,9.0)$ & & $71.37(\mathrm{~d})$ & \\
\hline G-5 & $3.10-3.15(1 \mathrm{H}, \mathrm{m})$ & & $77.84(d)$ & \\
\hline G-6a & $3.61(1 \mathrm{H}, \mathrm{dd}, J=12.1,5.7)$ & & $62.49(\mathrm{t})$ & \\
\hline G-6b & $3.76(1 \mathrm{H}, \mathrm{dd}, J=11.9,2.4)$ & & & \\
\hline
\end{tabular}

$\delta$ in ppm from TMS, $J$ value in $\mathrm{Hz}$, r.t.

The structure of 1 was further established by 2D-NMR studies, particularly ${ }^{1} \mathrm{H}-{ }^{1} \mathrm{H}$ correlated spectroscopy (COSY), heteronuclear multiple quantum coherence (HMQC) and nuclear Overhauser enhancement and exchange spectroscopy (NOESY). Correlations in HMBC and NOESY revealed proton-carbon sequences from $\mathrm{H}-2^{\prime}$ to $\mathrm{C}-8$ and $\mathrm{G}-\mathrm{C}-1, \mathrm{H}-1^{\prime}$ to C-7, C-8 and C-9, respectively.

Compounds $\mathbf{2}$ and $\mathbf{3}$ are also simple coumarin glycosides. $\mathbf{2}$ and $\mathbf{3}$ were identified as apiosylskimmin ${ }^{28)}$ and hymexelsin ${ }^{29)}$ by comparison of their structural data with those of authentic samples.

Nonglycosidic simple coumarins $4,5,6$, and 7 were identified as umbelliferone, scopoletin, ${ }^{30)}$ isofraxidin, ${ }^{31)}$ and 8 -carboxy-7-hydroxy coumarin ${ }^{32}$ based on analysis of their physical and spectral data (MS, ${ }^{1} \mathrm{H}$ - and ${ }^{13} \mathrm{C}$-NMR spectra, ${ }^{1} \mathrm{H}-{ }^{1} \mathrm{H}$ COSY, HMQC, HMBC experiments) and by comparison with literature values.

This is the first reported identification of simple coumarin (7) as a constituent of plant materials.

\section{Experimental}

General Experimental Procedures Melting points were recorded using a Yanagimoto melting point apparatus without correction. UV spectra were recorded using Shimadzu $1600 .{ }^{1} \mathrm{H}$ - and ${ }^{13} \mathrm{C}-\mathrm{NMR}$ spectra were recorded on a JNM-LA 500 spectrometer and a JEOL AL 400. FAB-MS spectra were obtained using a JMS-DX 302 mass spectrometer. Optical rotations were determined for solutions in $\mathrm{MeOH}$ using a JASCO DIP-140 polarimeter.

Plant Material Peucedanum praeruptorum DunN. (Umbelliferae) was collected from Ringan Town, Zhejiang Province, P. R. China, in August 2000 during the flowering season. A voucher specimen was identified by Prof. Xiao Luwei at Zhejiang College of Traditional Chinese Medicine and deposited at the herbarium of Meiji Pharmaceutical University.

Extraction and Isolation Pulverized roots of Peucedanum praeruptorum $(2.75 \mathrm{~kg})$ were extracted with water under reflux to give $638.6 \mathrm{~g}$ of extract. The water extract ( $638 \mathrm{~g}$ ) was fractionated with Diaion HP-20 and then HW-40F to give seven fractions, followed by HPLC with ODS (C18) and/or Develosil (C30) using a $\mathrm{MeOH}-\mathrm{H}_{2} \mathrm{O}$ solvent system to yield $\mathbf{1}(98.6 \mathrm{mg}), 2$ (87.7 mg), $3(6.3 \mathrm{mg}), \mathbf{4}(15.5 \mathrm{mg}), \mathbf{5}(9.7 \mathrm{mg}), \mathbf{6}(3.9 \mathrm{mg})$ and $7(23.4 \mathrm{mg})$.
Praeroside VI (1) White powder; $\mathrm{mp} 130.5-132.5^{\circ} \mathrm{C}$ (decomp.), $[\alpha]_{\mathrm{D}}^{25}$ $+1.0^{\circ}(c=1.0, \mathrm{MeOH})$. UV $(\mathrm{MeOH}): \lambda_{\max }(\log \varepsilon): 326.0 \mathrm{~nm}(3.70)$ $\left(\mathrm{MeOH}+\mathrm{CH}_{3} \mathrm{CO}_{2} \mathrm{Na}\right) 376.5 \mathrm{~nm}$. EI-MS $(70 \mathrm{eV}): \mathrm{m} / z$ (rel. int., \%): $426\left(\mathrm{M}^{+}\right.$, 1), 408 (2), 264 (26), 247 (38), 246 (34), 229 (29), 205 (64), 176 (100), 175 (86). HR-EI-MS: $m / z$ : 426.1525 (Calcd for $\mathrm{C}_{20} \mathrm{H}_{26} \mathrm{O}_{10}, 426.1526$ ). ${ }^{1} \mathrm{H}-$ and ${ }^{13}$ C-NMR: see Table 1.

Enzymatic Hydrolysis of 1 Enzymatic hydrolysis of $1(20 \mathrm{mg})$ with cellulase $(20 \mathrm{mg})$ in $\mathrm{H}_{2} \mathrm{O}(5 \mathrm{ml})$ at $40^{\circ} \mathrm{C}$ under stirring for $2 \mathrm{~d}$ afforded an aglycone (1a) and sugar moiety. 1a $(4.5 \mathrm{mg})$ was isolated from $n$-BuOH soluble part of reaction mixture.

(2'R)-7-Hydroxy-8-(2', 3'-dihydroxy-3'-methylbutyl)-coumarin (1a) White powder; $\mathrm{mp} 135.0-137.0^{\circ} \mathrm{C}$ (decomp.); $[\alpha]_{\mathrm{D}}^{25}+46.0^{\circ} \quad(c=0.50$, $\mathrm{MeOH})$.

Apiosylskimmin (2) White powder; $\mathrm{mp} 197.0-198.0^{\circ} \mathrm{C}$ (decomp.); $[\alpha]_{\mathrm{D}}^{25}-69.5^{\circ}\left(c=1.0\right.$, pyridine). UV (MeOH): $\lambda_{\max }(\log \varepsilon): 319.0 \mathrm{~nm}(4.04) ;$ FAB-MS: $m / z: 457[\mathrm{M}+\mathrm{H}]^{+}, 325[\mathrm{M}-\mathrm{api}+\mathrm{H}]^{+}, 163[\mathrm{M}-(\mathrm{api}+\mathrm{glc})+\mathrm{H}]^{+}$.

Hymexelsin (3) White powder; $[\alpha]_{\mathrm{D}}^{25}-104.4^{\circ}(c=0.50$, EtOH $)$; UV $(\mathrm{MeOH}): \lambda_{\max }(\log \varepsilon): 337.5 \mathrm{~nm}(3.75), 291.0 \mathrm{~nm}(3.68) ;$ FAB-MS: $m / z$ : 485 $[\mathrm{M}-\mathrm{H}]^{-}$

Umbelliferone (4) Colorless needles; $\mathrm{mp} 226.0-227.5^{\circ} \mathrm{C}$ (decomp.); UV (MeOH): $\lambda_{\max }(\log \varepsilon): 325.5 \mathrm{~nm}(3.88)$. EI-MS $(70 \mathrm{eV}): \mathrm{m} / z$ (rel. int., \%): $162\left(\mathrm{M}^{+}, 100\right), 134(89), 105(15), 78(16), 77(8)$.

Scopoletin (5) Colorless needles; $\mathrm{mp} 202.0-203.0^{\circ} \mathrm{C}$ (decomp.); UV $(\mathrm{MeOH}): \lambda_{\max }(\log \varepsilon): 344.0 \mathrm{~nm}(4.14), 297.5 \mathrm{~nm}(3.86), 253.5 \mathrm{~nm}(3.86)$. EI-MS (70 eV): $m / z$ (rel. int., \%): $192\left(\mathrm{M}^{+}, 100\right), 177$ (54), 164 (21), 149 (35), 135 (1), 121 (11).

Isofraxidin (6) Colorless needles; $\mathrm{mp} 149.0-150.0^{\circ} \mathrm{C}$ (decomp.); UV $(\mathrm{MeOH}): \lambda_{\max }(\log \varepsilon): 342.0 \mathrm{~nm}$ (3.74). EI-MS $(70 \mathrm{eV}): \mathrm{m} / z$ (rel. int., \%): $222\left(\mathrm{M}^{+}, 100\right), 207$ (25), 194 (15), 179 (18), 161 (5), 151 (10), 149 (4), 133 (5), 123 (13), 108 (7), $113(100)$.

8-Carboxy-7-hydroxy Coumarin (7) White powder; mp $210^{\circ} \mathrm{C}$ (decomp.); UV (MeOH): $\lambda_{\max }(\log \varepsilon): 333.0 \mathrm{~nm}(4.03), 258.0 \mathrm{~nm}$ (3.50). IR: $v^{\mathrm{KBr}}{ }_{\max } 3600-2600,1730,1715,1610,1505,1410,1360,1250,1230$ $840 \mathrm{~cm}^{-1}$. EI-MS (70 eV): $\mathrm{m} / z$ (rel. int., \%): $206\left(\mathrm{M}^{+}, 7\right), 188(23), 162(97)$, 160 (19), 134 (100), 132 (6), 105 (18), 78 (20), 77 (11).

Acknowledgement The authors are grateful to medical doctor Qian Xiao Hua at Ringan Town Hospital, Zhejiang Province, P. R. China, for support in the collection of Bai-Hua Qianhu, Peucedanum praeruptorum.

\section{References and Notes}

1) Part XXVI: Okada Y., Ishii H., Zhang Y., Baba M., Okuyama T., Phar- 
maceut. Biol., to be published.

2) Present address: Food Science \& Technology Research Group, Nichirei Foods Inc., Japan.

3) Okuyama T., Shibata S., Planta Med., 42, 89-96 (1981).

4) Kozawa T., Sakai K., Uchida M., Okuyama T., Shibata S., J. Pharm. Pharmacol., 33, 317-320 (1981).

5) Okuyama T., Sakakibara I., Shibata S., Syoyakugaku-Zasshi, 35, 331339 (1981).

6) Sakakibara I., Okuyama T., Shibata S., Planta Med., 44, 199-203 (1982).

7) Sakakibara I., Okuyama T., Shibata S., Planta Med., 50, 117-120 (1984).

8) Asahara T., Sakakibara I., Okuyama T., Shibata S., Planta Med., 50, $488-492(1984)$

9) Kawasaki C., Okuyama T., Shibata S., Iitaka Y., Planta Med., 50, 492-496 (1984)

10) Matano Y., Okuyama T., Shibata S., Hoson M., Kawada T., Osada H., Noguchi T., Planta Med., 52, 135-138 (1986).

11) Nishino H., Nishino A., Okuyama T., Shibata S., J. Kyoto Pref. Univ. Med., 96, 391-394 (1987).

12) Takata M., Okuyama T., Shibata S., Planta Med., 54, 323-327 (1988).

13) Okuyama T., Takata M., Shibata S., Planta Med., 55, 64-67 (1989).

14) Shibata S., Okuyama T., Abstr. Chin. Med., 3, 214-230 (1989).

15) Takata M., Shibata S., Okuyama T., Planta Med., 56, 307-311 (1990).

16) Takata M., Shibata S., Okuyama T., Planta Med., 56, 133 (1990).

17) Nishino H., Okuyama T., Takata M., Shibata S., Tokuda H., Takayasu
J., Hasegawa T., Nishino A., Ueyama H., Iwashima T., Carcinogenesis, 11, 1557-1561 (1990).

18) Chang T.-H., Li J.-M., Sun X.-D., Yu Y.-.F, Feng W.-Y., Hao L.-Y., Wang Y.-P. W., Zhang K.-Y., Okuyama T., Shoyakugaku Zasshi, 47, 279-282 (1993).

19) Chang T.-H., Adachi H., Mori N., Saito I., Okuyama T., Eur. J. Pharmacol., 258, 77—84 (1994).

20) Fulan G., Wanbao, J. Xinhua Z., Zhao N., Okuyama T., J. China Medical University, 23, 549-552 (1994).

21) Mizuno A., Okada Y., Nishino H., Okuyama T., J. Tradit. Med., 11, $220-224$ (1994).

22) Zhao N.-C., Jin W.-B., Zhang X.-H., Guan F.-L., Sun Y.-B., Adachi H., Okuyama T., Biol. Pharm. Bull., 22, 984-987 (1999).

23) Chang H., Okada Y., Okuyama T., Tu P., Magn. Reson. Chem., 45, $611-614$ (2007).

24) Chang H.-T., Okada Y., Ma T.-G., Okuyama T., Tu P.-F., J. Asian Nat. Prod. Res., 10, 577-581 (2008).

25) Lemmich J., Phytochemistry, 38, 427-432 (1995).

26) Ceccherelli P., J. Nat. Prod., 53, 536-538 (1990).

27) Derek R. B., Chem. Commun., 2002, 3070-3071 (2002).

28) Satyanarayana P., Phytochemistry, 24, 1862-1863 (1985)

29) Rao P. S., Asheervadam Y., J. Nat. Prod., 51, 959-961 (1988).

30) Ito C., Furukawa H., Chem. Pharm. Bull., 35, 4277-4286 (1987).

31) Tsukamoto H., Hisada S., Nishibe S., Chem. Pharm. Bull., 33, 40694073 (1985).

32) Soine T. O., Erhardt P., Zheleva A., Bubeva-Ivanova L., Mahandru M. M., J. Pharm. Sci., 62, 1879-1880 (1973). 